\title{
Several Differentiation Formulas of Special Functions. Part IV
}

\author{
Bo Li \\ Qingdao University of Science \\ and Technology \\ China
}

\author{
Peng Wang \\ Qingdao University of Science \\ and Technology \\ China
}

Summary. In this article, we give several differentiation formulas of special and composite functions including trigonometric function, polynomial function and logarithmic function.

MML identifier: FDIFF_8, version: 7.8.03 4.75.958

The notation and terminology used here are introduced in the following papers: [13], [15], [1], [16], [2], [4], [10], [11], [17], [5], [14], [12], [3], [7], [6], [9], and [8].

For simplicity, we adopt the following convention: $x, a, b, c$ denote real numbers, $n$ denotes a natural number, $Z$ denotes an open subset of $\mathbb{R}$, and $f$, $f_{1}, f_{2}$ denote partial functions from $\mathbb{R}$ to $\mathbb{R}$.

Next we state a number of propositions:

(1) If $x \in \operatorname{dom}($ the function tan), then (the function $\cos )(x) \neq 0$.

(2) If $x \in \operatorname{dom}$ (the function cot), then (the function $\sin )(x) \neq 0$.

(3) If $Z \subseteq \operatorname{dom}\left(\frac{f_{1}}{f_{2}}\right)$, then for every $x$ such that $x \in Z$ holds $\left(\frac{f_{1}}{f_{2}}\right)(x)_{\mathbb{Z}}^{n}=\frac{f_{1}(x)_{Z}^{n}}{f_{2}(x)_{\mathbb{Z}}^{n}}$.

(4) Suppose $Z \subseteq \operatorname{dom}\left(\frac{f_{1}}{f_{2}}\right)$ and for every $x$ such that $x \in Z$ holds $f_{1}(x)=$ $x+a$ and $f_{2}(x)=x-b$. Then $\frac{f_{1}}{f_{2}}$ is differentiable on $Z$ and for every $x$ such that $x \in Z$ holds $\left(\frac{f_{1}}{f_{2}}\right)_{\uparrow Z}^{\prime}(x)=\frac{-a-b}{(x-b)^{2}}$.

(5) Suppose $Z \subseteq \operatorname{dom}\left((\right.$ the function $\left.\ln ) \cdot \frac{1}{f}\right)$ and for every $x$ such that $x \in Z$ holds $f(x)=x$. Then (the function $\ln ) \cdot \frac{1}{f}$ is differentiable on $Z$ and for every $x$ such that $x \in Z$ holds ((the function $\left.\ln ) \cdot \frac{1}{f}\right)_{{ }^{\prime} Z}^{\prime}(x)=-\frac{1}{x}$.

(6) Suppose $Z \subseteq \operatorname{dom}(($ the function tan $) \cdot f)$ and for every $x$ such that $x \in Z$ holds $f(x)=a \cdot x+b$. Then 
(i) (the function tan) $\cdot f$ is differentiable on $Z$, and

(ii) for every $x$ such that $x \in Z$ holds ((the function $\tan ) \cdot f)^{\prime}{ }_{{ }_{Z}}(x)=$ $\frac{a}{\text { (the function } \cos )(a \cdot x+b)^{2}}$.

(7) Suppose $Z \subseteq \operatorname{dom}(($ the function cot $) \cdot f)$ and for every $x$ such that $x \in Z$ holds $f(x)=a \cdot x+b$. Then

(i) (the function cot) $\cdot f$ is differentiable on $Z$, and

(ii) for every $x$ such that $x \in Z$ holds ((the function cot) $\cdot f)^{\prime}{ }_{\mid Z}(x)=$ $-\frac{a}{\text { (the function } \sin )(a \cdot x+b)^{2}}$.

(8) Suppose $Z \subseteq \operatorname{dom}\left((\right.$ the function $\left.\tan ) \cdot \frac{1}{f}\right)$ and for every $x$ such that $x \in Z$ holds $f(x)=x$. Then

(i) (the function tan) $\frac{1}{f}$ is differentiable on $Z$, and

(ii) for every $x$ such that $x \in Z$ holds ((the function tan) $\left.\cdot \frac{1}{f}\right)_{{ }_{1}}^{\prime}(x)=$ $-\frac{1}{x^{2} \cdot(\text { the function } \cos )\left(\frac{1}{x}\right)^{2}}$.

(9) Suppose $Z \subseteq \operatorname{dom}\left((\right.$ the function cot $\left.) \cdot \frac{1}{f}\right)$ and for every $x$ such that $x \in Z$ holds $f(x)=x$. Then

(i) (the function cot) $\cdot \frac{1}{f}$ is differentiable on $Z$, and

(ii) for every $x$ such that $x \in Z$ holds ((the function cot) $\left.\cdot \frac{1}{f}\right)_{{ }_{1 Z}^{\prime}}^{\prime}(x)=$ $\frac{1}{x^{2} \cdot(\text { the function } \sin )\left(\frac{1}{x}\right)^{2}}$.

(10) Suppose $Z \subseteq \operatorname{dom}\left((\right.$ the function $\left.\tan ) \cdot\left(f_{1}+c f_{2}\right)\right)$ and $f_{2}={ }_{\mathbb{Z}}^{2}$ and for every $x$ such that $x \in Z$ holds $f_{1}(x)=a+b \cdot x$. Then

(i) (the function tan) $\cdot\left(f_{1}+c f_{2}\right)$ is differentiable on $Z$, and

(ii) for every $x$ such that $x \in Z$ holds ((the function tan) $\left.\cdot\left(f_{1}+c f_{2}\right)\right)^{\prime}{ }_{\Gamma Z}(x)=$ $\frac{b+2 \cdot c \cdot x}{\text { (the function } \cos )\left(a+b \cdot x+c \cdot x^{2}\right)^{2}}$.

(11) Suppose $Z \subseteq \operatorname{dom}\left((\right.$ the function cot $\left.) \cdot\left(f_{1}+c f_{2}\right)\right)$ and $f_{2}={ }_{\mathbb{Z}}^{2}$ and for every $x$ such that $x \in Z$ holds $f_{1}(x)=a+b \cdot x$. Then

(i) (the function cot) $\cdot\left(f_{1}+c f_{2}\right)$ is differentiable on $Z$, and

(ii) for every $x$ such that $x \in Z$ holds ((the function cot) $\left.\cdot\left(f_{1}+c f_{2}\right)\right)_{\lceil Z}^{\prime}(x)=$ $-\frac{b+2 \cdot c \cdot x}{(\text { the function } \sin )\left(a+b \cdot x+c \cdot x^{2}\right)^{2}}$.

(12) Suppose $Z \subseteq \operatorname{dom}(($ the function tan) $\cdot($ the function exp)). Then

(i) (the function tan) $\cdot$ (the function $\exp$ ) is differentiable on $Z$, and

(ii) for every $x$ such that $x \in Z$ holds ((the function tan) -(the function $\exp ))^{\prime}(x)=\frac{(\text { the function exp) }(x)}{(\text { the function cos) }(\text { (the function } \exp )(x))^{2}}$.

(13) Suppose $Z \subseteq \operatorname{dom}(($ the function cot) $\cdot($ the function exp)). Then

(i) (the function cot) (the function $\exp$ ) is differentiable on $Z$, and

(ii) for every $x$ such that $x \in Z$ holds ((the function cot) -(the function $\exp ))^{\prime}{ }_{Y Z}(x)=-\frac{(\text { the function exp })(x)}{\left(\text { the function sin) }((\text { the function } \exp )(x))^{2}\right.}$.

(14) Suppose $Z \subseteq \operatorname{dom}(($ the function $\tan ) \cdot($ the function $\ln ))$. Then

(i) (the function $\tan ) \cdot($ the function $\ln$ ) is differentiable on $Z$, and 
(ii) for every $x$ such that $x \in Z$ holds ((the function tan) $\cdot$ (the function $\ln ))^{\prime}(x)=\frac{1}{x \cdot(\text { the function } \cos )((\text { the function } \ln )(x))^{2}}$.

(15) Suppose $Z \subseteq \operatorname{dom}(($ the function cot) $\cdot($ the function $\ln ))$. Then

(i) (the function cot) (the function $\ln$ ) is differentiable on $Z$, and

(ii) for every $x$ such that $x \in Z$ holds ((the function cot) $\cdot$ (the function $\ln ))_{{ }^{\prime} Z}^{\prime}(x)=-\frac{1}{x \cdot(\text { the function sin)((the function } \ln )(x))^{2}}$.

(16) Suppose $Z \subseteq \operatorname{dom}(($ the function $\exp ) \cdot($ the function tan $))$. Then

(i) (the function exp) (the function tan) is differentiable on $Z$, and

(ii) for every $x$ such that $x \in Z$ holds ((the function exp) ·(the function $\tan ))_{{ }^{\prime} Z}^{\prime}(x)=\frac{(\text { the function exp })((\text { the function } \tan )(x))}{(\text { the function } \cos )(x)^{2}}$.

(17) Suppose $Z \subseteq \operatorname{dom}(($ the function $\exp ) \cdot($ the function cot)). Then

(i) (the function exp) -(the function cot) is differentiable on $Z$, and

(ii) for every $x$ such that $x \in Z$ holds ((the function $\exp$ ) (the function cot $))^{\prime}{ }_{Z}(x)=-\frac{(\text { the function exp })((\text { the function } \cot )(x))}{(\text { the function } \sin )(x)^{2}}$.

(18) Suppose $Z \subseteq \operatorname{dom}(($ the function $\ln ) \cdot($ the function tan $))$. Then

(i) (the function $\ln$ ) $\cdot$ (the function $\tan$ ) is differentiable on $Z$, and

(ii) for every $x$ such that $x \in Z$ holds ((the function $\ln$ ) $\cdot$ (the function $\tan ))^{\prime}{ }_{i Z}(x)=\frac{1}{(\text { the function } \cos )(x) \cdot(\text { the function } \sin )(x)}$.

(19) Suppose $Z \subseteq \operatorname{dom}(($ the function $\ln ) \cdot($ the function cot $))$. Then

(i) (the function $\ln ) \cdot($ the function cot) is differentiable on $Z$, and

(ii) for every $x$ such that $x \in Z$ holds ((the function $\ln$ ) ·(the function cot $))^{\prime}{ }(x)=-\frac{1}{(\text { the function } \sin )(x) \cdot(\text { the function } \cos )(x)}$.

(20) Suppose $Z \subseteq \operatorname{dom}\left(\left(\begin{array}{l}n \\ \mathbb{Z}\end{array}\right) \cdot(\right.$ the function tan $\left.)\right)$ and $1 \leq n$. Then

(i) $\left(\begin{array}{l}n \\ \mathbb{Z}\end{array}\right) \cdot($ the function $\tan )$ is differentiable on $Z$, and

(ii) for every $x$ such that $x \in Z$ holds $\left(\left(\begin{array}{l}n \\ \mathbb{Z}\end{array}\right) \cdot(\text { the function } \tan )\right)^{\prime}{ }_{\mid Z}(x)=$ $\frac{n \cdot(\text { the function } \sin )(x)_{\mathbb{Z}}^{n-1}}{(\text { the function } \cos )(x)_{\mathbb{Z}}^{n+1}}$.

(21) Suppose $Z \subseteq \operatorname{dom}\left(\left(\begin{array}{l}n \\ \mathbb{Z}\end{array}\right) \cdot(\right.$ the function cot)) and $1 \leq n$. Then

(i) $\left(\begin{array}{l}n \\ \mathbb{Z}\end{array}\right) \cdot($ the function cot) is differentiable on $Z$, and

(ii) for every $x$ such that $x \in Z$ holds $\left(\left(\begin{array}{l}n \\ \mathbb{Z}\end{array}\right) \cdot(\text { the function } \cot )\right)^{\prime}{ }_{Z}(x)=$ $-\frac{n \cdot(\text { the function } \cos )(x)_{\mathbb{Z}}^{n-1}}{(\text { the function } \sin )(x)_{\mathbb{Z}}^{n+1}}$.

(22) Suppose that

(i) $Z \subseteq \operatorname{dom}\left((\right.$ the function $\left.\tan )+\frac{1}{\text { the function cos }}\right)$, and

(ii) for every $x$ such that $x \in Z$ holds $1+($ the function $\sin )(x) \neq 0$ and $1-($ the function $\sin )(x) \neq 0$.

Then

(iii) (the function $\tan )+\frac{1}{\text { the function cos }}$ is differentiable on $Z$, and

(iv) for every $x$ such that $x \in Z$ holds $\left((\text { the function tan })+\frac{1}{\text { the function cos }}\right)_{\mid Z}^{\prime}(x)=$ $\frac{1}{1-(\text { the function } \sin )(x)}$. 
(23) Suppose that

(i) $Z \subseteq \operatorname{dom}\left((\right.$ the function $\left.\tan )-\frac{1}{\text { the function cos }}\right)$, and

(ii) for every $x$ such that $x \in Z$ holds $1-($ the function $\sin )(x) \neq 0$ and $1+($ the function $\sin )(x) \neq 0$.

Then

(iii) (the function tan) $-\frac{1}{\text { the function } \cos }$ is differentiable on $Z$, and

(iv) for every $x$ such that $x \in Z$ holds $\left((\text { the function tan })-\frac{1}{\text { the function cos }}\right)_{\uparrow Z}^{\prime}(x)=$ $\frac{1}{1+(\text { the function } \sin )(x)}$.

(24) Suppose $Z \subseteq \operatorname{dom}\left((\right.$ the function $\left.\tan )-\operatorname{id}_{Z}\right)$. Then

(i) (the function $\tan )-\mathrm{id}_{Z}$ is differentiable on $Z$, and

(ii) for every $x$ such that $x \in Z$ holds ((the function $\left.\tan )-\mathrm{id}_{Z}\right)_{{ }^{\prime} Z}^{\prime}(x)=$ $\frac{\text { (the function } \sin )(x)^{2}}{\text { (the function } \cos )(x)^{2}}$.

(25) Suppose $Z \subseteq \operatorname{dom}\left(-\right.$ the function $\left.\cot -\mathrm{id}_{Z}\right)$. Then

(i) -the function cot $-\mathrm{id}_{Z}$ is differentiable on $Z$, and

(ii) for every $x$ such that $x \in Z$ holds (-the function $\left.\cot -\operatorname{id}_{Z}\right)_{\mid Z}^{\prime}(x)=$ $\frac{(\text { the function } \cos )(x)^{2}}{\text { (the function } \sin )(x)^{2}}$.

(26) Suppose $Z \subseteq \operatorname{dom}\left(\frac{1}{a}((\right.$ the function $\left.\tan ) \cdot f)-\operatorname{id}_{Z}\right)$ and for every $x$ such that $x \in Z$ holds $f(x)=a \cdot x$ and $a \neq 0$. Then

(i) $\frac{1}{a}$ ((the function $\left.\left.\tan \right) \cdot f\right)-\operatorname{id}_{Z}$ is differentiable on $Z$, and

(ii) for every $x$ such that $x \in Z$ holds $\left(\frac{1}{a}((\right.$ the function $\tan ) \cdot f)-$ $\left.\operatorname{id}_{Z}\right)^{\prime}(x)=\frac{(\text { the function } \sin )(a \cdot x)^{2}}{(\text { the function } \cos )(a \cdot x)^{2}}$

(27) Suppose $Z \subseteq \operatorname{dom}\left(\left(-\frac{1}{a}\right)((\right.$ the function $\left.\cot ) \cdot f)-\operatorname{id}_{Z}\right)$ and for every $x$ such that $x \in Z$ holds $f(x)=a \cdot x$ and $a \neq 0$. Then

(i) $\quad\left(-\frac{1}{a}\right)(($ the function cot $) \cdot f)-\mathrm{id}_{Z}$ is differentiable on $Z$, and

(ii) for every $x$ such that $x \in Z$ holds $\left(\left(-\frac{1}{a}\right)((\right.$ the function cot $) \cdot f)-$ $\left.\operatorname{id}_{Z}\right)_{\lceil Z}^{\prime}(x)=\frac{(\text { the function } \cos )(a \cdot x)^{2}}{(\text { the function } \sin )(a \cdot x)^{2}}$

(28) Suppose $Z \subseteq \operatorname{dom}(f$ (the function $\tan ))$ and for every $x$ such that $x \in Z$ holds $f(x)=a \cdot x+b$. Then

(i) $\quad f$ (the function tan) is differentiable on $Z$, and

(ii) for every $x$ such that $x \in Z$ holds $(f$ (the function $\tan ))^{\prime}{ }_{\mid Z}(x)=$ $\frac{a \cdot(\text { the function } \sin )(x)}{(\text { the function } \cos )(x)}+\frac{a \cdot x+b}{\text { (the function } \cos )(x)^{2}}$.

(29) Suppose $Z \subseteq \operatorname{dom}(f$ (the function cot)) and for every $x$ such that $x \in Z$ holds $f(x)=a \cdot x+b$. Then

(i) $\quad f$ (the function cot) is differentiable on $Z$, and

(ii) for every $x$ such that $x \in Z$ holds $(f \text { (the function cot) })_{1 Z}^{\prime}(x)=$ $\frac{a \cdot(\text { the function } \cos )(x)}{(\text { the function } \sin )(x)}-\frac{a \cdot x+b}{\text { (the function } \sin )(x)^{2}}$.

(30) Suppose $Z \subseteq \operatorname{dom}(($ the function $\exp )$ (the function tan)). Then

(i) (the function exp) (the function tan) is differentiable on $Z$, and 
(ii) for every $x$ such that $x \in Z$ holds ((the function exp) (the function $\tan ))^{\prime}{ }_{i}(x)=\frac{(\text { the function exp })(x) \cdot(\text { the function } \sin )(x)}{(\text { the function cos) }(x)}+\frac{(\text { the function exp })(x)}{(\text { the function } \cos )(x)^{2}}$.

(31) Suppose $Z \subseteq \operatorname{dom}(($ the function $\exp$ ) (the function cot)). Then

(i) (the function exp) (the function cot) is differentiable on $Z$, and

(ii) for every $x$ such that $x \in Z$ holds ((the function exp) (the function cot) $)^{\prime}{ }_{i Z}(x)=\frac{(\text { the function } \exp )(x) \cdot(\text { the function } \cos )(x)}{(\text { the function } \sin )(x)}-\frac{(\text { the function } \exp )(x)}{\text { (the function } \sin )(x)^{2}}$.

(32) Suppose $Z \subseteq \operatorname{dom}(($ the function $\ln$ ) (the function tan)). Then

(i) (the function $\ln$ ) (the function tan) is differentiable on $Z$, and

(ii) for every $x$ such that $x \in Z$ holds ((the function $\ln$ ) (the function $\tan ))_{\lceil Z}^{\prime}(x)=\frac{\frac{(\text { the function } \sin )(x)}{(\text { the function } \cos )(x)}}{x}+\frac{(\text { the function } \ln )(x)}{(\text { the function } \cos )(x)^{2}}$.

(33) Suppose $Z \subseteq \operatorname{dom}(($ the function $\ln )$ (the function cot)). Then

(i) (the function ln) (the function cot) is differentiable on $Z$, and

(ii) for every $x$ such that $x \in Z$ holds ((the function $\ln$ ) (the function cot $))_{{ }^{\prime}}^{\prime}(x)=\frac{\frac{(\text { the function } \cos )(x)}{\text { (the function } \sin )(x)}}{x}-\frac{(\text { the function } \ln )(x)}{(\text { the function } \sin )(x)^{2}}$.

(34) Suppose $Z \subseteq \operatorname{dom}\left(\frac{1}{f}\right.$ (the function $\tan$ )) and for every $x$ such that $x \in Z$ holds $f(x)=x$. Then

(i) $\frac{1}{f}$ (the function tan) is differentiable on $Z$, and

(ii) for every $x$ such that $x \in Z$ holds $\left(\frac{1}{f}\right.$ (the function $\left.\left.\tan \right)\right)^{\prime}{ }_{{ }^{Z}}(x)=$ $-\frac{\frac{(\text { the function } \sin )(x)}{(\text { the function } \cos )(x)}}{x^{2}}+\frac{\frac{1}{x}}{(\text { the function } \cos )(x)^{2}}$.

(35) Suppose $Z \subseteq \operatorname{dom}\left(\frac{1}{f}\right.$ (the function cot)) and for every $x$ such that $x \in Z$ holds $f(x)=x$. Then

(i) $\frac{1}{f}$ (the function cot) is differentiable on $Z$, and

(ii) for every $x$ such that $x \in Z$ holds $\left(\frac{1}{f}\right.$ (the function $\left.\left.\cot \right)\right)_{{ }^{\prime}}^{\prime}(x)=$ $-\frac{\frac{(\text { the function } \cos )(x)}{(\text { the function } \sin )(x)}}{x^{2}}-\frac{\frac{1}{x}}{(\text { the function } \sin )(x)^{2}}$.

\section{REFERENCES}

[1] Grzegorz Bancerek. The ordinal numbers. Formalized Mathematics, 1(1):91-96, 1990.

[2] Czesław Byliński. Partial functions. Formalized Mathematics, 1(2):357-367, 1990.

[3] Krzysztof Hryniewiecki. Basic properties of real numbers. Formalized Mathematics, 1(1):35-40, 1990.

[4] Jarosław Kotowicz. Partial functions from a domain to a domain. Formalized Mathematics, 1(4):697-702, 1990.

[5] Jarosław Kotowicz. Partial functions from a domain to the set of real numbers. Formalized Mathematics, 1(4):703-709, 1990.

[6] Jarosław Kotowicz. Real sequences and basic operations on them. Formalized Mathematics, 1(2):269-272, 1990.

[7] Rafal Kwiatek. Factorial and Newton coefficients. Formalized Mathematics, 1(5):887-890, 1990.

[8] Konrad Raczkowski. Integer and rational exponents. Formalized Mathematics, 2(1):125130, 1991.

[9] Konrad Raczkowski and Paweł Sadowski. Real function differentiability. Formalized Mathematics, 1(4):797-801, 1990. 
[10] Konrad Raczkowski and Paweł Sadowski. Topological properties of subsets in real numbers. Formalized Mathematics, 1(4):777-780, 1990.

[11] Yasunari Shidama. The Taylor expansions. Formalized Mathematics, 12(2):195-200, 2004.

[12] Andrzej Trybulec. Subsets of complex numbers. To appear in Formalized Mathematics.

[13] Andrzej Trybulec. Tarski Grothendieck set theory. Formalized Mathematics, 1(1):9-11, 1990.

[14] Andrzej Trybulec and Czesław Byliński. Some properties of real numbers. Formalized Mathematics, 1(3):445-449, 1990.

[15] Zinaida Trybulec. Properties of subsets. Formalized Mathematics, 1(1):67-71, 1990.

[16] Edmund Woronowicz. Relations defined on sets. Formalized Mathematics, 1(1):181-186, 1990.

[17] Yuguang Yang and Yasunari Shidama. Trigonometric functions and existence of circle ratio. Formalized Mathematics, 7(2):255-263, 1998.

Received September 29, 2006 